PROCEEDINGS OF THE AMERICAN MATHEMATICAL SOCIETY

Volume 124, Number 9, September 1996

\title{
CONORMAL DIFFERENTIAL FORMS OF AN ANALYTIC GERM
}

\author{
ROBERT GASSLER
}

(Communicated by Eric Friedlander)

\begin{abstract}
A differential form vanishing on the tangent space at smooth points of a reduced embedded analytic germ is called conormal. To prove that a conormal one-form of a hypersurface vanishes at its singularities, we state a Bertini-type theorem.
\end{abstract}

\section{INTRODUCTION}

The purpose of this paper is to study properties of differential forms which vanish on the tangent space of an analytic germ at its regular points. We will call them conormal to the germ. They form a differential ideal whose properties will be studied in section 2 . In section 4 we state our main result, saying that conormal one-forms of a hypersurface vanish at its singularities. For the proof we need two ingredients: a Bertini-type theorem, which we prove in section 3, and a result about tangential vector fields which are isomorphic to conormal $(n-1)$-forms. The last section gives some examples of our forms.

Conormal forms have been studied before under the name torsion differentials. See $[Y]$ for the definition and more references.

\section{Notation}

The germs of holomorphic functions on $\mathbb{C}^{n}$ at 0 will be denoted by $\mathcal{O}$, and holomorphic differential forms by $\Omega$, and holomorphic vector fields by $\mathbb{D}$. All our analytic germs will be embedded and reduced. We will identify them with their well-chosen representatives. In the following, $X \subseteq\left(\mathbb{C}^{n}, 0\right)$ will be an analytic germ. The germ of regular points of $X$ is denoted by $X^{\text {reg }}$, the singular locus by $\operatorname{Sing} X$, and the Lie Algebra of tangential vector fields by $\mathbb{D}_{X}$.

The Zariski tangent space of $X$ at 0 is denoted by $\mathrm{T}_{0} X$. In the following we will use the identification $\mathrm{T}_{0} \mathbb{C}^{n} \cong \mathbb{C}^{n}$ induced by the canonical chart on $\mathbb{C}^{n}$ without mention.

The tangent plane space $\tau X$ of $X$ is the closure of the tangent bundle $\mathrm{T} X^{\mathrm{reg}} \subseteq$ $\left(\mathbb{C}^{n}, 0\right) \times \mathbb{C}^{n}$. If $X$ is of pure dimension $r$ the closure of $\left\{\left(p, \mathrm{~T}_{p} X\right), p \in X^{\mathrm{reg}}\right\} \subseteq$ $\left(\mathbb{C}^{n}, 0\right) \times \mathbb{G}^{n-1, r-1}$ will also be called the tangent plane space $\tau^{*} X$ of $X$. The fibers of these bundles over a point $p$ will be denoted by the subindex $p$. The tangent plane space is compatible with the inclusion of analytic germs: for analytic germs

Received by the editors February 23, 1995.

1991 Mathematics Subject Classification. Primary 14B99, 14K20, 14F10.

(C)1996 American Mathematical Society 
$X \subseteq Y \subseteq\left(\mathbb{C}^{n}, 0\right)$ we have $\tau X \subseteq \tau Y$, as proved by Whitney [Wh, p. 548], as an application of the existence of Whitney stratifications.

\section{CONORMAL FORMS OF ANALYTIC GERMS}

Definition (Conormal differential forms). A differential form $\omega \in \Omega$ is called conormal to $X$ if it vanishes on $\mathrm{T}^{\mathrm{reg}}$. This means each $i$-homogeneous part of $\omega$ vanishes on $\left(\mathrm{T}_{p} X\right)^{i}$ for $p \in X^{\mathrm{reg}}$.

The set of all conormal differential forms of $X$ is denoted by $\mathbb{F}_{X}$, and the set of $k$-homogeneous conormal forms by $\mathbb{F}_{X}^{k}$.

Proposition (Properties of conormal forms). (i) $\mathbb{F}_{X}$ is a differential ideal in $\Omega$.

(ii) Let $X=\mathrm{V}\left(f_{1}, \ldots, f_{m}\right)$ be a complete intersection. A differential form $\omega \in \Omega$ is conormal to $X$ iff $\omega \wedge d f_{1} \wedge \cdots \wedge d f_{m}$ vanishes on $X$.

(iii) The ideal of $X$ equals $\mathbb{F}_{X}^{0}$.

(iv) A differential form is conormal to $X$ iff it annihilates the tangent plane space $\tau X$.

(v) An analytic germ $Y \subseteq\left(\mathbb{C}^{n}, 0\right)$ is contained in $X$ iff $\mathbb{F}_{X} \subseteq \mathbb{F}_{Y}$.

(vi) Let $X_{1}, \ldots, X_{m}$ be the irreducible components of $X$. Then $\mathbb{F}_{X}=\mathbb{F}_{X_{1}} \cap \cdots \cap$ $\mathbb{F}_{X_{m}}$.

(vii) The sequence

$$
0 \rightarrow \mathbb{F}_{X}^{0} \stackrel{d}{\rightarrow} \mathbb{F}_{X}^{1} \stackrel{d}{\rightarrow} \ldots \stackrel{d}{\rightarrow} \mathbb{F}_{X}^{n} \rightarrow 0
$$

is exact at $\mathbb{F}_{X}^{0}$ and $\mathbb{F}_{X}^{1}$.

Proof. (i) The pull-back of differential forms under the embedding $i: X^{\text {reg }} \hookrightarrow$ $\left(\mathbb{C}^{n}, 0\right)$ is a homomorphism of differential algebras. $\mathbb{F}_{X}$ is its kernel, hence a differential-ideal.

(ii) We must prove the assertion only at regular points, because $d f_{1} \wedge \cdots \wedge d f_{m}$ vanishes on Sing $X$. Furthermore, $\omega$ can be assumed to be $r$-homogeneous, because of the linearity of the exterior product. For $r=0$ the assertion is obvious, since $d f_{1} \wedge \cdots \wedge d f_{m}$ does not vanish on $X$. For $r>0$ and $p \in X^{\text {reg }}$ we know that the linear forms $d f_{i}(p)$ are linearly independent. Hence, we can extend them to a basis $\left(l_{i}\right)$ of $\mathrm{T}_{p}^{*} X$. Now $\omega(p)$ annihilates $\mathrm{T}_{p} X=\bigcap_{1}^{m} \operatorname{Ker} l_{i}$ iff it is an element of the ideal generated by $l_{1}, \ldots, l_{m}$ in the $\mathbb{C}$-algebra $\wedge \mathrm{T}_{p}^{*} \mathbb{C}^{n}$. This is equivalent to $\omega(p) \wedge l_{1} \wedge \cdots \wedge l_{m}=\left(\omega \wedge d f_{1} \wedge \cdots \wedge d f_{m}\right)(p)=0$.

(iii) This is clear by the definition of $\mathbb{F}_{X}^{0}$.

(iv) Follows from the definition of $\tau X$.

(v) For $Y \subseteq X$ we have $\tau Y \subseteq \tau X$. With (iv) we get $\mathbb{F}_{X} \subseteq \mathbb{F}_{Y}$. If $\mathbb{F}_{X} \subseteq \mathbb{F}_{Y}$ we have with (iii)

$$
\mathrm{I}_{X}=\mathbb{F}_{X}^{0} \subseteq \mathbb{F}_{Y}^{0}=\mathrm{I}_{Y} .
$$

(vi) The inclusion $\subseteq$ follows from (v). On the other hand every $\omega \in \mathbb{F}_{X_{1}} \cap \cdots \cap$ $\mathbb{F}_{X_{m}}$ vanishes on the tangent space of $X$ at a regular point, as it is also a regular point in one irreducible component.

(vii) The only thing to show is that for every $\omega \in \mathbb{F}_{X}^{1}$ with $d \omega=0$ there is a $g \in \mathbb{F}_{X}^{0}=\mathrm{I}_{X}$ with $d g=\omega$. The Lemma of Poincaré gives us the existence of a function $f \in \mathcal{O}$ with $\omega=d f$. We now show $g:=f-f(0) \in \mathrm{I}_{X}$. For each point $p \in X^{\text {reg }}$ we can find a continuous, piecewise differentiable path $\gamma:[0,1] \rightarrow X$ connecting 0 and $p$, which intersects Sing $X$ in a finite number of points, as $X^{\text {reg }}$ 
is a manifold, dense in $X$, and has only a finite number of connected components. (The existence of such a path can be seen by desingularizing $X$.) We now have

$$
g(p)=f(p)-f(0)=\int_{\gamma} d f=\int_{0}^{1} \omega(\gamma(t)) \cdot \dot{\gamma}(t)=0,
$$

because $\dot{\gamma}(t) \in \mathrm{T}_{\gamma(t)} X \subseteq \operatorname{Ker} \omega(\gamma(t))$ for all but a finite number of times.

\section{INTERSECTIONS OF HYPERPLANES AND HYPERSURFACES}

In this section we will prove a special type of Bertini's Theorem, which could also be derived from the results of Flenner [Fl] and Jouanalou [J]. Here, an idea of Teissier [T, Lemma 1.1] will be further developed to give a very geometric proof of the theorem for our special case. We will also consider non-reduced analytic germs in this section.

The following proposition collects results proved by Teissier [T, Lemma 1.1] and Whitney [Wh, Lemma 6.1]. For the Grassmannian $\mathbb{G}^{n-1, n-2}$ of hyperplanes in $\mathbb{C}^{n}$ we will write $\mathbb{G}$. A hyperplane will be called transversal to $X$ if it is transversal to $X^{\text {reg. }}$.

Proposition (Transversal hyperplanes). Let $X \subseteq\left(\mathbb{C}^{n}, 0\right)$ be of pure dimension greater than 0 . The intersection of $X$ and a transversal hyperplane has dimension $\operatorname{dim} X-1$. If $X$ is smooth, so is $H \cap X$. The non-empty set $W=\{H \in \mathbb{G}$ : $H$ is transversal to every element of $\left.\tau_{0}^{*} X\right\}$ is dense in $\mathbb{G}$. Each of its elements is transversal to $X$.

Theorem (Theorem of Bertini for analytic germs). Let $X$ be a reduced hypersurface in $\left(\mathbb{C}^{n}, 0\right), n \geq 3$. For each hyperplane $H \in \mathbb{G}$ transversal to $X$ and $\operatorname{Sing} X$, the intersection $H \cap X$ is reduced and

$$
\operatorname{Sing}(X \cap H)=H \cap \operatorname{Sing} X \text {. }
$$

By the above proposition the set of these hyperplanes is dense in $\mathbb{G}$.

Proof. For every $H \in \mathbb{G}$ transversal to $X$ we have $H \cap X^{\text {reg }} \subseteq(H \cap X)^{\text {reg }}$ by the above proposition. On the other hand, we know that $H \cap \operatorname{Sing} X \subseteq \operatorname{Sing}(X \cap H)$.

If the hypersurface $H \cap X \subseteq H$ were not reduced, at least one of its irreducible components would be contained in its singular locus. Hence, Sing $X \cap H$ would be one-codimensional in $H$. But, it has codimension at least two, which is obvious if Sing $X$ equals 0 , and otherwise follows from the above proposition.

\section{Conormal one-Forms of a hypersurface}

We now study the behavior of one-forms conormal to a hypersurface at its singularities. In the following $X \subseteq\left(\mathbb{C}^{n}, 0\right)$ is a hypersurface, whose ideal is generated by $f \in \mathcal{O}$.

Proposition (Conormal $(n-1)$-forms of a hypersurface). A conormal $(n-1)-$ form of $X$ not vanishing at 0 induces a decomposition $X \cong X^{\prime} \times(\mathbb{C}, 0)$, where $X^{\prime} \subseteq\left(\mathbb{C}^{n-1}, 0\right)$ is an analytic germ.

Proof. The $\mathcal{O}$-linear map $D: \Omega^{n-1} \rightarrow \mathbb{D}, \omega \mapsto(f \mapsto \phi(\omega \wedge d f))$, with $\phi$ the isomorphism mapping $d x_{1} \wedge \cdots \wedge d x_{n}$ to 1 , is a bijection and maps $\mathbb{F}_{X}^{n-1}$ to $\mathbb{D}_{X}$, as $\left.(D(\omega) \cdot f)\right|_{X}=\phi\left(\left.\omega \wedge d f\right|_{X}\right)=0$ for $\omega$ conormal to $X$. Applying the Theorem of Rossi to the image under $D$ of a form conormal to $X$ and not vanishing at 0 give us the decomposition [R, Theorem 3.2]. 
Remark. Note that we used the natural orientation and metric on $\mathbb{C}^{n}$ to define the bijection $\phi: \Omega^{n-1} \rightarrow \mathcal{O}$.

Theorem (Conormal one-forms of a hypersurface). Each conormal one-form of $X$ vanishes on $\operatorname{Sing} X$.

Proof. $\operatorname{dim} X=1$ : A one-form not vanishing in 0 induces the decomposition $X \cong$ $(\mathbb{C}, 0) \times X^{\prime}$, where $X^{\prime}$ is analytic, as proved in the above proposition. It is obvious that such a decomposition only exists if $X$ is regular.

Induction on $\operatorname{dim} X$ : Suppose the theorem is proved for hypersurfaces $X \subseteq$ $\left(\mathbb{C}^{n-1}, 0\right)$, and look at a singular point which can be considered to be 0 without loss of generality. By the Theorem of Bertini there exist two hyperplanes $H_{1}, H_{2} \subseteq$ $\left(\mathbb{C}^{n}, 0\right)$ transversal to each other such that $H_{i} \cap X$ is reduced and $\operatorname{Sing}\left(H_{i} \cap X\right)=$ $H_{i} \cap \operatorname{Sing} X$. Consider now a conormal 1-form $\omega$ of $X$. By (v) of the proposition of properties of conormal forms, $\left.\omega\right|_{\mathrm{T} H_{i}}$ is also conormal to the hypersurface $H_{i} \cap X \subseteq$ $H_{i}$. For this case our theorem is supposed to be proved, so $\omega$ vanishes on $\mathrm{T}_{0} H_{i}$. These two tangential spaces span $\mathbb{C}^{n}$, and we get $\omega(0)=0$.

\section{EXAMPLES}

In this section we will use the following result proved by Vetter [V] and Lebelt [L]. Furthermore, $\mathbb{F}_{X}^{k}$ will be called trivial if it equals the $\mathcal{O}$-module of $k$-homogeneous forms of the differential ideal generated by $\mathrm{I}_{X}$ in $\Omega$.

Theorem (Trivial conormal forms). Let $X \subseteq\left(\mathbb{C}^{n}, 0\right)$ be a complete intersection. For $k \leq \operatorname{dim} X$ the following are equivalent:

(i) $X$ is regular in codimension $k$.

(ii) $\mathbb{F}_{X}^{k}$ is trivial.

1. $X=\mathrm{V}\left(x_{1}, \ldots, x_{m}\right) \subseteq\left(\mathbb{C}^{n}, 0\right)$. The differential ideal $\mathbb{F}_{X}$ of $\Omega$ is generated by $\left(x_{1}, \ldots, x_{m}\right)$. This can be proved by an easy computation using the characterization of conormal forms, but it also follows directly from the above theorem.

2. $X=\mathrm{V}\left(x^{3}-y z\right) \cdot \mathbb{F}_{X}^{1}$ is trivial, but there is a non-trivial conormal 2-form $\omega=x d y \wedge d z+3 z d x \wedge d y$, which generates, together with the trivial forms, $\mathbb{F}_{X}^{2}$ as an $\mathcal{O}$-module.

3. $X=\mathrm{V}\left(z^{2}-x y^{2}\right)$, the Whitney-umbrella. $\mathbb{F}_{X}$ is generated by $z^{2}-x y^{2}, y z d x+$ $2 x z d y-2 x y d z$, and $y d x \wedge d z-z d x \wedge d y$.

4. $\quad X=\mathrm{V}(x z-y t)$. Both $\mathbb{F}_{X}^{1}$ and $\mathbb{F}_{X}^{2}$ are trivial. A non-trivial 3 -form is $\omega=(x d y-y d x) \wedge d z \wedge d t$.

\section{ACKNOWLEDGMent}

This work was finished during my stay in Valladolid, Spain, financed by an ERASMUS scholarship. I want to thank the members of the Department of Algebra, Geometry, and Topology for their hospitality, and of course Herwig Hauser for supervising this work and spending his time discussing problems with me. I thank Bernard Teissier for pointing out references $[\mathrm{Fl}]$ and $[\mathrm{J}]$ to me.

\section{REFERENCES}

[Fl] H. Flenner, Die Sätze von Bertini für lokale Ringe, Math. Ann. 229 (1977), 97-111. MR $\mathbf{5 7 : 3 1 1}$

[J] J.P. Jouanalou, Théorèmes de Bertini et Applicationes, Birkhäuser, 1983. MR 86b:13007 
[L] K. Lebelt, Torsion äußerer Potenzen von Moduln der homologischen Dimension 1, Math. Ann 211 (1974), 183-197. MR 51:8092

[R] H. Rossi, Vector fields on Analytic Spaces, Ann. of Math. 78 (1963), 455-467. MR 29:277

[T] B. Teissier, Cycles evanescents, sections planes et condition de Whitney, Asterisque 7-8 (1973), 285-362. MR 51:10682

[V] U. Vetter, Äußere Potenzen von Differentialmoduln reduzierter vollständiger Durchschnitte, Manuscripta Math. 2 (1970), 67-75. MR 42:255

[Wh] H. Whitney, Tangents to an Analytic Variety, Ann. Math. 81 (1965), 496-549. MR 33:745

[Y] S. S.-T. Yau, Various Numerical Invariants for Isolated Singularities, Amer. J. Math. 104 (1982), 1063-1100. MR 84b:32012

Department of Mathematics, 567 Lake, Northeastern University, Boston, MassaCHUSETTS 02115

E-mail address: gassler@neu.edu 